\title{
Gauge Instantons from Open Strings
}

\author{
M. Frau ${ }^{1}$, A. Lerda ${ }^{2,1} *$ \\ ${ }^{1}$ Dipartimento di Fisica Teorica, Università di Torino \\ and I.N.F.N., sezione di Torino \\ via P. Giuria 1, I-10125 Torino, Italy \\ 2 Dipartimento di Scienze e Tecnologie Avanzate \\ Università del Piemonte Orientale, I-15100 Alessandria, Italy
}

\begin{abstract}
In this contribution we describe how to obtain instanton effects in four dimensional gauge theories by computing string scattering amplitudes in $\mathrm{D} 3 / \mathrm{D}(-1)$ brane systems. In particular we show that the disks with mixed boundary conditions, which are typical of the $\mathrm{D} 3 / \mathrm{D}(-1)$ system, are the sources for the classical instanton solution.
\end{abstract}

\section{Introduction}

In the last few years remarkable progress in the study of (supersymmetric) field theories has been achieved by embedding them into string theory and analyzing the infinite tension limit $\alpha^{\prime} \rightarrow 0$. In a perturbative framework, one typically considers string scattering amplitudes on a Riemann surface $\Sigma$ of a given topology

$$
A_{N}=\phi_{1} \cdots \phi_{N} \int_{\Sigma}\left\langle\mathcal{V}_{\phi_{1}} \cdots \mathcal{V}_{\phi_{N}}\right\rangle_{\Sigma}
$$

where the vertex operators $\mathcal{V}_{\phi_{i}}$ describe the emission of the fields $\phi_{i}$ from the world-sheet, and $\langle\cdots\rangle_{\Sigma}$ denotes the expectation value with respect to the vacuum represented by $\Sigma$. For closed strings the simplest world-sheets are spheres, while for open strings they are disks. On these world-sheets there are no tadpoles: in fact one has

$$
\left\langle\mathcal{V}_{\phi_{\text {closed }}}\right\rangle_{\text {sphere }}=0
$$

for any closed string field $\phi_{\text {closed, }}$, and

$$
\left\langle\mathcal{V}_{\phi_{\text {open }}}\right\rangle_{\text {disk }}=0
$$

for any open string field $\phi_{\text {open }}$. Thus, spheres and disks are appropriate to describe the trivial vacua around which the ordinary perturbation theory is performed, but are clearly inadequate to describe classical non-perturbative backgrounds.

After the discovery of D-branes [1] this perspective has drastically changed and nowadays several non-perturbative features of field theory can be described in string theory. The key observation is that, despite their non-perturbative nature, the D-branes admit a

*corresponding author E-mail: lerda@to.infn.it 
perturbative description in terms of closed strings whose left and right movers are suitably identified. This amounts to insert a boundary on the world-sheet so that the simplest topology for closed strings in the presence of a $\mathrm{D} p$ brane is that of disks with $(p+1)$ longitudinal (or Neumann) and $(9-p)$ transverse (or Dirichlet) boundary conditions. As a consequence of the identifications between left and right movers, on these disks there are closed-string tadpoles, i.e. in general

$$
\left\langle\mathcal{V}_{\phi_{\text {closed }}}\right\rangle_{\operatorname{disk}_{p}} \neq 0
$$

A $\mathrm{D} p$ brane can also be represented by a boundary state $|\mathrm{D} p\rangle$, which is a nonperturbative state of the closed string that inserts a boundary on the world-sheet and enforces on it the appropriate boundary conditions (for a review on the boundary state formalism, see for example Ref. [2]). If we denote by $\left|\phi_{\text {closed }}\right\rangle$ the physical state associated to the vertex operator $\mathcal{V}_{\phi_{\text {closed }}}$, we have

$$
\left\langle\phi_{\text {closed }} \mid \mathrm{D} p\right\rangle=\left\langle\mathcal{V}_{\phi_{\text {closed }}}\right\rangle_{\operatorname{disk}_{p}} .
$$

Thus, the boundary state, or equivalently its corresponding disk, is a classical source for the various fields of the closed string spectrum. In particular, it is a source for the massless fields (like the graviton, or the Ramond-Ramond $(p+1)$-form potential) which can acquire a non-trivial profile and describe a classical background characterized by a non-trivial geometry and a non-vanishing Ramond-Ramond charge. The precise relation between such a background and the boundary state has been established in Ref. [3], where it has been shown that if one multiplies the massless tadpoles of $|\mathrm{D} p\rangle$ by free propagators and then takes the Fourier transform, one gets the leading terms in the large distance expansion of the classical $\mathrm{D} p$-brane solution. These arguments prove that, in order to describe closed strings in the non-perturbative D-brane background it is necessary to modify the boundary conditions of the string coordinates and, at the lowest order, consider disks instead of spheres.

A natural question is whether this kind of approach can be generalized to open strings, and in particular whether one can describe in this way the instantons of four dimensional gauge theories. A positive answer to this question has been given in Ref. [4, and in this contribution we briefly review the main results of that paper. The crucial point is that the instantons of (supersymmetric) gauge theories are non-perturbative configurations which admit a perturbative description within the realm of open string theory. Thus, they are the analogue for open strings of what the supergravity $p$-branes with RamondRamond charge are for closed strings. In our analysis, a key role is played again by the D-branes. For definiteness, let us consider a stack of $N$ D3 branes of Type IIB string theory which support on their world-volume a four-dimensional $\mathcal{N}=4$ super Yang-Mills theory with gauge group $\mathrm{U}(N)^{1}$. Then, in order to describe instantons of this gauge theory with topological charge $k$, one has to introduce $k \mathrm{D}(-1)$ branes (D-instantons) and thus consider a $\mathrm{D} 3 / \mathrm{D}(-1)$ brane system [5]. In this system, besides the open strings stretching between two D3 branes and representing the usual perturbative gauge degrees of freedom, there are also open strings with at least one end-point on the D-instantons. These strings describe non-dynamical degrees of freedom which can be interpreted as the moduli of the gauge (super)instantons in the ADHM construction (for a review on this construction and its realization using D-branes, see for instance Ref. [6]).

In Ref. 4] we have shown that this well-known D-brane description is not only an efficient book-keeping device to account for the multiplicities of the various instanton

${ }^{1}$ or $\mathrm{SU}(N)$ if we disregard the center of mass. 


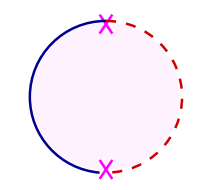

Figure 1: The simplest mixed disk with two-boundary changing operators indicated by the two crosses. The solid line represents the D3 boundary while the dashed line represents the $\mathrm{D}(-1)$ boundary.

moduli, but is also a powerful tool to extract from string theory a detailed information on the gauge instantons. The important point is that in the $\mathrm{D} 3 / \mathrm{D}(-1)$ system the presence of different boundary conditions for the open strings implies the existence of disks whose boundary is divided into different portions lying either on the D3 or on the D $(-1)$ branes (see Fig. 1). These disks, which we call mixed disks, are characterized by the insertion of at least two vertex operators associated to excitations of strings that stretch between a D3 and a $\mathrm{D}(-1)$ brane (or vice-versa), and depend on the moduli that accompany these mixed vertex operators. Due to the change in the boundary conditions caused by the mixed operators, in general one may expect that

$$
\left\langle\mathcal{V}_{\phi_{\text {open }}}\right\rangle_{\text {mixed disk }} \neq 0
$$

In Ref. 4] we have shown that this expectation is indeed correct. In particular the massless fields of the $\mathcal{N}=4$ gauge vector multiplet propagating on the D3 branes have non-trivial tadpoles on mixed disks, and their Fourier transform (after including a propagator) yields precisely the classical instanton solution of the $\mathrm{SU}(\mathrm{N})$ gauge theory in the singular gauge. In the next sections we briefly review the main features of the $\mathrm{D} 3 / \mathrm{D}(-1)$ brane system, show how to obtain the classical instanton profile from open string amplitudes on mixed disks, and finally present our conclusions.

\section{The D3/D(-1) system}

As we mentioned above, the $k$ instanton sector of a four-dimensional $\mathcal{N}=4 \mathrm{SYM}$ theory with gauge group $\mathrm{SU}(N)$ can be described by a bound state of $N$ D3 and $k \mathrm{D}(-1)$ branes [5]. In the $\mathrm{D} 3 / \mathrm{D}(-1)$ system the string coordinates $X^{\mathcal{M}}$ and $\psi^{\mathcal{M}}(\mathcal{M}=1, \ldots, 10)$ obey different boundary conditions depending on the type of boundary. Specifically, on the $\mathrm{D}(-1)$ brane we have Dirichlet boundary conditions in all directions, while on the D3 brane the longitudinal fields $X^{\mu}$ and $\psi^{\mu}(\mu=1,2,3,4)$ satisfy Neumann boundary conditions, and the transverse fields $X^{a}$ and $\psi^{a}(a=5, \ldots, 10)$ obey Dirichlet boundary conditions. These conditions, in turn, imply suitable reflection rules for the spin fields, which can be found in Ref. [4] ${ }^{2}$. For our present purposes it is enough to specify the spectrum of excitations of the open strings with at least one end-point on the D-instantons. Let us first consider the strings that have both ends on the $\mathrm{D}(-1)$ branes: in the NS sector the physical excitations are $a_{\mu}$ and $\chi^{a}$, whose corresponding vertex operators are

$$
V_{a}(z)=a^{\mu} \psi_{\mu}(z) \mathrm{e}^{-\phi(z)} \quad, \quad V_{\chi}(z)=\chi^{a} \psi_{a}(z) \mathrm{e}^{-\phi(z)}
$$

where $\phi$ is the chiral boson of the superghost bosonization. In the $\mathrm{R}$ sector we find sixteen fermionic moduli which are conventionally denoted by $M^{\alpha A}$ and $\lambda_{\dot{\alpha} A}$, and correspond to

\footnotetext{
${ }^{2}$ Here we recall that the presence of the D3 branes breaks $\mathrm{SO}(10)$ into $\mathrm{SO}(4) \times \mathrm{SO}(6)$, so that the 10-dimensional spin fields decompose into $S_{\alpha} S_{A}$ and $S^{\dot{\alpha}} S^{A}$ where $S_{\alpha}\left(S^{\dot{\alpha}}\right)$ are $\mathrm{SO}(4)$ Weyl spinors of positive (negative) chirality, and $S^{A}\left(S_{A}\right)$ are $\mathrm{SO}(6)$ Weyl spinors of positive (negative) chirality which transform in the fundamental (anti-fundamental) representation of $\mathrm{SU}(4) \sim \mathrm{SO}(6)$.
} 
the following vertex operators

$$
V_{M}(z)=M^{\alpha A} S_{\alpha}(z) S_{A}(z) \mathrm{e}^{-\frac{1}{2} \phi(z)} \quad, \quad V_{\lambda}(z)=\lambda_{\dot{\alpha} A} S^{\dot{\alpha}}(z) S^{A}(z) \mathrm{e}^{-\frac{1}{2} \phi(z)} .
$$

Let us now consider the open strings that start on a D3 and end on a D $(-1)$ brane, or viceversa. These strings are characterized by the fact that four directions (the longitudinal ones to the D3 brane) have mixed boundary conditions. Thus, in the NS sector the four fields $\psi^{\mu}$ have integer-mode expansions with zero-modes that represent the $\mathrm{SO}(4)$ Clifford algebra and the corresponding physical excitations are organized in two bosonic Weyl spinors of $\mathrm{SO}(4)$. These are denoted by $w_{\dot{\alpha}}$ and $\bar{w}_{\dot{\alpha}}$ respectively, and are described by the following vertex operators

$$
V_{w}(z)=w_{\dot{\alpha}} \Delta(z) S^{\dot{\alpha}}(z) \mathrm{e}^{-\phi(z)} \quad, \quad V_{\bar{w}}(z)=\bar{w}_{\dot{\alpha}} \bar{\Delta}(z) S^{\dot{\alpha}}(z) \mathrm{e}^{-\phi(z)} .
$$

Here $\Delta(z)$ and $\bar{\Delta}(z)$ are the bosonic twist and anti-twist fields with conformal dimension $1 / 4$, that change the boundary conditions of the $X^{\mu}$ coordinates from Neumann to Dirichlet and vice-versa by introducing a cut in the world-sheet. In the $\mathrm{R}$ sector the fields $\psi^{\mu}$ have, instead, half-integer mode expansions so that there are fermionic zero-modes only in the six common transverse directions. Thus, the physical excitations of this sector form two fermionic Weyl spinors of $\mathrm{SO}(6)$ which are denoted by $\mu^{A}$ and $\bar{\mu}^{A}$ respectively, and correspond to the following vertex operators

$$
V_{\mu}(z)=\mu^{A} \Delta(z) S_{A}(z) \mathrm{e}^{-\frac{1}{2} \phi(z)} \quad, \quad V_{\bar{\mu}}(z)=\bar{\mu}^{A} \bar{\Delta}(z) S_{A}(z) \mathrm{e}^{-\frac{1}{2} \phi(z)} .
$$

A systematic analysis [4 shows that, in the limit $\alpha^{\prime} \rightarrow 0$, the scattering amplitudes involving the above vertex operators give rise to the following "action"

$$
\begin{aligned}
S= & \operatorname{tr}_{k}\left\{-\left[a^{\mu}, \chi^{a}\right]^{2}+\chi_{a} \bar{w}_{\dot{\alpha}} w^{\dot{\alpha}} \chi^{a}+\frac{\mathrm{i}}{2} \bar{\Sigma}_{A B}^{a} \bar{\mu}^{A} \mu^{B} \chi_{a}-\frac{\mathrm{i}}{4} \bar{\Sigma}_{A B}^{a} M^{\alpha A}\left[\chi_{a}, M_{\alpha}{ }^{B}\right]\right. \\
& \left.+\mathrm{i}\left(\bar{\mu}^{A} w_{\dot{\alpha}}+\bar{w}_{\dot{\alpha}} \mu^{A}+\left[M^{\alpha A}, a_{\alpha \dot{\alpha}}\right]\right) \lambda^{\dot{\alpha}}{ }_{A}-\mathrm{i}\left(w_{\dot{\alpha}}\left(\tau^{c}\right)_{\dot{\beta}}^{\dot{\alpha}} \bar{w}^{\dot{\beta}}+\mathrm{i} \bar{\eta}_{\mu \nu}^{c}\left[a^{\mu}, a^{\nu}\right]\right) D_{c}\right\}
\end{aligned}
$$

where $\operatorname{tr}_{k}$ means trace over $\mathrm{U}(k), \bar{\Sigma}^{a}$ are the Dirac matrices of $\mathrm{SO}(6), \bar{\eta}^{c}(c=1,2,3)$ are the anti-self dual 't Hooft symbols, and $\tau^{c}$ are the Pauli matrices. In (11) there appear also three auxiliary fields $D_{c}$ whose string representation is provided by the following vertex operators [4]

$$
V_{D}(z)=\frac{1}{2} D_{c} \bar{\eta}_{\mu \nu}^{c} \psi^{\nu}(z) \psi^{\mu}(z)
$$

As is well known [6], by simply taking $\mathrm{e}^{-S}$ one obtains the measure on the instanton moduli space, while by varying $S$ with respect to $D_{c}$ and $\lambda^{\dot{\alpha}}{ }_{A}$ one easily derives the bosonic and fermionic ADHM constraints.

\section{The instanton solution from mixed disks}

We now show that the mixed disks of the $\mathrm{D} 3 / \mathrm{D}(-1)$ brane system (see Fig. 11) are the source for the instanton background of the super Yang-Mills theory, and that the classical instanton profile can be obtained from open string amplitudes. For simplicity we will discuss only the case of instanton number $k=1$ in a $\mathrm{SU}(2)$ gauge theory, but no substantial changes occur in our analysis if one considers higher values of $k$ and other 


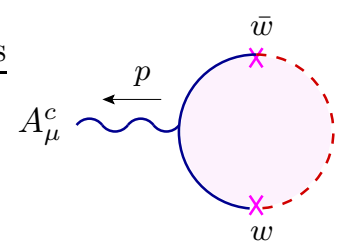

Figure 2: The mixed disk that describes the emission of a gauge vector field $A_{\mu}^{c}$ with momentum $p$ represented by the outgoing wavy line.

gauge groups (see Ref. [4] for these extensions). Let us then consider the emission of the $\mathrm{SU}(2)$ gauge vector field $A_{\mu}^{c}$ from a mixed disk. The simplest diagram which can contribute to this process contains two boundary changing operators $V_{\bar{w}}$ and $V_{w}$ and no other moduli, and is shown in Fig. 2. The amplitude associated to this diagram is

$$
\left\langle\mathcal{V}_{A_{\mu}^{c}(-p)}\right\rangle_{\text {mixed disk }} \equiv\left\langle V_{\bar{w}} \mathcal{V}_{A_{\mu}^{c}(-p)} V_{w}\right\rangle=\mathcal{A}_{\mu}^{c}(p ; \bar{w}, w)
$$

where the gluon vertex operator $\mathcal{V}_{A_{\mu}^{c}(-p)}$ is with outgoing momentum $p$ and without polarization, so that the amplitude carries the structure of an emitted gauge vector field. The evaluation of the amplitude (13) is quite straightforward and the result is (4]

$$
\mathcal{A}_{\mu}^{c}(p ; \bar{w}, w)=\mathrm{i} \rho^{2} p^{\nu} \bar{\eta}_{\nu \mu}^{c} \mathrm{e}^{-\mathrm{i} p \cdot x_{0}}
$$

where we have defined $\bar{w}^{\dot{\alpha}} w_{\dot{\alpha}}=2 \rho^{2}$ and denoted by $x_{0}$ the location of the D-instanton inside the world-volume of the D3 branes. By taking the Fourier transform of (14), after inserting a free propagator [3], we obtain

$$
\mathcal{A}_{\mu}^{c}(x)=\int \frac{d^{4} p}{(2 \pi)^{2}} \frac{1}{p^{2}} \mathcal{A}_{\mu}^{c}(p ; \bar{w}, w) \mathrm{e}^{\mathrm{i} p \cdot x}=\frac{2 \rho^{2} \bar{\eta}_{\mu \nu}^{c}\left(x-x_{0}\right)^{\nu}}{\left(x-x_{0}\right)^{4}} .
$$

In (15) we recognize the leading term in the large distance expansion (i.e. $\left|x-x_{0}\right|>>\rho$ ) of the $\mathrm{SU}(2)$ instanton with center $x_{0}$ and size $\rho$ in the singular gauge, namely

$$
\mathcal{A}_{\mu}^{c}(x)=\frac{2 \rho^{2} \bar{\eta}_{\mu \nu}^{c}\left(x-x_{0}\right)^{\nu}}{\left(x-x_{0}\right)^{2}\left[\left(x-x_{0}\right)^{2}+\rho^{2}\right]} \simeq \frac{2 \rho^{2} \bar{\eta}_{\mu \nu}^{c}\left(x-x_{0}\right)^{\nu}}{\left(x-x_{0}\right)^{4}}\left(1-\frac{\rho^{2}}{\left(x-x_{0}\right)^{2}}+\ldots\right) .
$$

This result shows that a mixed disk, like that of Fig. 2, is the source for the classical gauge instanton. Note that the amplitude (13) is a 3-point function from the point of view of the two dimensional conformal field theory on the string world sheet, but is a 1-point function from the point of view of the four-dimensional gauge theory on the D3 branes. In fact, the two boundary changing operators $V_{\bar{w}}$ and $V_{w}$ that appear in (13) just describe non-dynamical parameters on which the background depends. Furthermore, the fact that the gluon field (15) is in the singular gauge is not surprising, because in our set-up the gauge instanton is produced by a $\mathrm{D}(-1)$ brane which is a point-like object inside the $\mathrm{D} 3$ brane world-volume. Thus it is natural that the gauge connection exhibits a singularity at the location $x_{0}$ of the D-instanton.

An obvious question at this point is whether also the subleading terms in the large distance expansion (16) have a direct interpretation in string theory. Since such terms contain higher powers of $\rho^{2} \sim \bar{w}^{\dot{\alpha}} w_{\dot{\alpha}}$, one expects that they are associated to mixed disks with more insertions of boundary changing operators. This expectation has been explicitly confirmed in Ref. [4], so that one can conclude that mixed disks with the emission 
of a gauge vector field do reproduce the complete $k=1 \mathrm{SU}(2)$ instanton solution. As mentioned above, this result can be extended to other instanton numbers (and other gauge groups) in a straightforward way.

\section{Conclusions}

In this contribution we have considered, for simplicity, only the emission of gluons from mixed disks; however, this approach can be easily generalized to study the emission of the complete vector supermultiplet and recover in this way the full superinstanton solutions of the $\mathcal{N}=4$ super Yang-Mills theory [4. Furthermore, this analysis can be extended without difficulties also to $\mathrm{D} 3 / \mathrm{D}(-1)$ brane systems in orbifold backgrounds that reduce the supersymmetry to $\mathcal{N}=2$ or $\mathcal{N}=1$, as well as to brane systems in the presence of constant NS-NS or R-R background fields.

In conclusion we have have shown that the mixed disks are the sources for the gauge (super)instantons, and thus, they are the appropriate world-sheets one has to consider in order to compute instanton contributions to correlation functions within string theory. We believe that this result helps to clarify the analysis and the prescriptions presented for example in Ref. [7 and also provides the conceptual bridge necessary to relate the D-instanton techniques of string theory to the instanton calculus of field theory.

Acknowledgements: We would like to deeply thank our coauthors: M. Billò, F. Fucito, A. Liccardo and I. Pesando. This work is partially supported by the European Commission RTN programme HPRN-CT-2000-00131 and by MIUR under contract 2003-023852.

\section{References}

[1] J. Polchinski, Phys. Rev. Lett. 75, 4724 (1995) arXiv:hep-th/9510017.

[2] P. Di Vecchia and A. Liccardo, "D branes in string theory. I" arXiv:hep-th/9912161; "D-branes in string theory. II" arXiv:hep-th/9912275.

[3] P. Di Vecchia, M. Frau, I. Pesando, S. Sciuto, A. Lerda and R. Russo, Nucl. Phys. B 507, 259 (1997) arXiv:hep-th/9707068; P. Di Vecchia, M. Frau, A. Lerda and A. Liccardo, Nucl. Phys. B 565, 397 (2000) arXiv:hep-th/9906214.

[4] M. Billò, M. Frau, I. Pesando, F. Fucito, A. Lerda and A. Liccardo, JHEP 0302, 045 (2003) arXiv:hep-th/0211250.

[5] E. Witten, Nucl. Phys. B 460, 335 (1996) arXiv:hep-th/9510135; M. R. Douglas, "Branes within branes" arXiv:hep-th/9512077; J. Geom. Phys. 28, 255 (1998) arXiv:hep-th/9604198.

[6] N. Dorey, T. J. Hollowood, V. V. Khoze and M. P. Mattis, Phys. Rept. 371, 231 (2002) arXiv:hep-th/0206063.

[7] M.B. Green and M. Gutperle, Nucl. Phys. B 498, 195 (1997) arXiv:hep-th/9701093; JHEP 9801, 005 (1998) arXiv:hep-th/9711107; JHEP 0002, 014 (2000) arXiv:hep-th/0002011. 